\title{
INELASTIC NEUTRON SCATTERING FROM ICE AND OTHER PROTON-CONTAINING SUBSTANCES
}

\author{
By Bruno DORner \\ (Institut Laue-Langevin, I 56X, Centre de Tri, 38042 Grenoble Cédex, France)
}

\begin{abstract}
A brief introduction to neutron scattering is given with special emphasis on the difference between coherent and incoherent contributions. The following report of recent work on ice contains the determination and verification of some phonon dispersion curves, and outlines the state of the art on diffuse scattering due to the proton disorder. Results on "two-dimensional ice" in copper formate tetrahydrate are of great interest as this system exhibits a phase transformation below which the protons are ordered. The study of rotational diffusion and of the tunnel splitting of the rotational ground state of molecules which contain protons is discussed in some detail. These investigations are now possible with very high energy resolution $\Delta E \approx 5 \times 10^{-8} \mathrm{eV}\left(4 \times 10^{-4} \mathrm{~cm}^{-1}\right.$ or $\left.12 \mathrm{MHz}\right)$.
\end{abstract}

RÉsumé. Diffusion inélastique des neutrons dans la glace ou dans d'autres substances contenant des protons. Une brève introduction est consacrée à la diffusion des neutrons et nous insistons plus particulièrement sur la différence entre les contributions cohérentes et incohérentes. Dans cet article relatif à des travaux récents sur la glace, nous présentons la détermination et la vérification de quelques courbes de dispersion par les phonons et dégageons les bases de la technique de dispersion diffuse due au désordre protonique. Des résultats correspondant à une "glace bidimensionnelle" dans le système cuivre-formate-tetrahydrate sont d'un grand intérêt car un tel système présente une transformation de phase au-dessous de laquelle les protons sont ordonnés. L'étude de la diffusion rotationnelle et du clivage par effet tunnel du niveau rotationnel fondamental des molécules dotées de protons, est discutée de façon quelque peu détaillée. Ce type d'investigation est maintenant possible avec une très haute résolution en énergie: $\Delta E \approx 5 \cdot 10^{-8} \mathrm{eV}\left(4^{\cdot} \cdot 10^{-4} \mathrm{~cm}^{-1}\right.$ ou $12 \mathrm{MHz}$ ).

Zusammenfassung. Inelastische Neutronenstreuung von Eis und anderen protonenhaltigen Substanzen. Es wird eine kurze Einführung in die Neutronenstreuung gegeben mit besonderem Hinweis auf den Unterschied zwischen kohärentem und inkohärentem Beitrag. Der folgende Bericht über neuere Arbeiten an Eis enthält die Bestimmung und Bestätigung von einigen Phononen-Dispersions-Kurven und zeigt den Stand der Untersuchungen der diffusen Streuung, die von der Unordnung der Protonen herrührt. Ergebnisse an "zwei-dimensionalem Eis" in Kupfer-Format-Tetrahydrat sind von grossem Interesse, da dieses System einen Phasenübergang aufweist, unterhalb dessen die Protonen geordnet sind. Untersuchungen der Rotationsdiffusion und der Tunnelaufspaltung des Rotationsgrundzustandes von Molekülen, die Protonen enthalten, werden im Einzelnen diskutiert. Diese Messungen sind neuerdings möglich mit sehr hoher Energieauflösung $\Delta E \approx 5 \cdot 10^{-8} \mathrm{eV}\left(4 \cdot 10^{-4} \mathrm{~cm}^{-1}\right.$ oder $\left.12 \mathrm{MHz}\right)$.

\section{INTRODUCTION}

One may say that neutron scattering is now a well-established technique. Nevertheless, in recent years the progress towards better resolution in instrumentation has been considerable. The Institut Laue-Langevin possesses one of the most sophisticated high-flux reactors in the world. The beam time on such instruments is much in demand by outside users.

As far as neutron scattering from ice is concerned there are not many new results to be reported. The reason is probably that ice is a very complicated substance and even with the best energy resolution available today, for example, the study of the dynamics of proton or deuteron diffusion in ice is still impossible.

In this paper I will give a brief introduction to diffuse and inelastic scattering (Dorner and Comès, 1977) explaining the coherent and incoherent components in the scattered intensity. Then I will present a summary of recent work on ice, and finally I would like to explain highresolution inelastic neutron scattering by giving two examples: (i) the microscopic mechanism of rotational diffusion in solid adamantane, and (ii) tunnelling between the rotational ground states of $\mathrm{NH}_{4}$ in $\mathrm{NH}_{4} \mathrm{ClO}_{4}$ at low temperature. I include this latter part because $\mathrm{I}$ expect that many participants at this conference are interested in the results of investigations into the positions and movements of protons within solids. 


\section{NEUTRON SCATTERING}

The intensity scattered with a momentum transfer $\hbar \mathbf{Q}$ is described by the scattering law $S(\mathbf{Q}) . S(\mathbf{Q})$ is the square of the sum of all amplitudes of the scattered waves from the $j$ nuclei,

$$
S(\mathbf{Q})=\left|\sum_{j} b_{j} \exp \left(-w_{j}\right) \exp \left(\mathbf{i} \mathbf{Q} \cdot\left(\mathbf{R}_{j}+\mathbf{U}_{j}\right)\right)\right|^{2},
$$

where $b_{j}$ and $\exp \left(-w_{j}\right)$ are the scattering length and the Debye-Waller factor of nucleus $j$. $\mathbf{R}_{j}+\mathbf{U}_{j}$ is the actual position of nucleus $j$. The summation is carried out over the entire crystal. If we assume a periodic structure (i.e. a single crystal), then $\mathbf{R}_{j}$ is the periodic position and $\mathbf{U}_{j}$ the displacement from it. In a periodic structure the sum over the crystal can be replaced by a sum over the crystalline unit cell.

If we expand Equation (I) for small $\mathbf{U}_{j}$ we obtain

$$
S(\mathbf{Q})=\mid \sum_{j} b_{j} \exp \left(-w_{j}+\left.\mathrm{i} \mathbf{Q} \cdot \mathbf{R}_{j}\right|^{2}+\left|\sum_{j} b_{j}\left(\mathbf{Q} \cdot \mathbf{U}_{j}\right) \exp \left(-w_{j}+\mathrm{i} \mathbf{Q} \cdot \mathbf{R}_{j}\right)\right|^{2} .\right.
$$

The summations are now carried out over the unit cell. Linear terms in $U$ vanish at $\langle\mathbf{U}\rangle=$ o. The first expression gives the Bragg intensities, which are localized as $\delta$-functions in reciprocal space. It may contain elastic incoherent scattering depending on the $b_{j}$ 's as discussed later. Our main interest lies in the second part which we call the diffuse scattering $S_{\mathrm{d}}(\mathbf{Q})$, because intensity may occur for every $\mathbf{Q}$ depending on the $\mathbf{U}$ 's.

Expanding Equation (2) there will be expressions $b_{l} b_{k}$ and $b_{j}{ }^{2}$. If different species of atoms are found at $l$ and $k$ in their particular symmetry positions within the unit cell, then there is no problem. But if different isotopes of the same element occupy sites $l$ and $k$, or if at $l$ the spin of the neutron is parallel to the spin of the nucleus and at $k$ antiparallel, then $b_{l}$ may be much different from $b_{k}$. Unfortunately the isotopes or spin configurations are usually randomly distributed over the sites. To avoid this difficulty we define a linearly averaged scattering length $b$ for each element and call it the coherent scattering length. We also define a quadratic average $\overline{b^{2}}$ and obtain the so-called incoherent cross-section $4 \pi\left(\overline{b^{2}}-\bar{b}^{2}\right)$ per element. The diffuse scattering then reads

$$
\begin{aligned}
S_{\mathbf{d}}(\mathbf{Q})=n \sum_{j}\left(\overline{b_{j}^{2}}-\bar{b}_{j}{ }^{2}\right)\left(\mathbf{Q} \cdot \mathbf{U}_{j}\right)^{2} \exp \left(-2 w_{j}\right)^{2}+ & \\
& +\left|\sum_{j} \bar{b}_{j}\left(\mathbf{Q} \cdot \mathbf{U}_{j}\right) \exp \left(-w_{j}+\mathbf{i} \mathbf{Q} \cdot \mathbf{R}_{j}\right)\right|^{2} .
\end{aligned}
$$

Here $n$ is the number of unit cells and again, the summation is carried out over the unit cells. The first term is the incoherent part of the diffuse scattering, being just the sum over the incoherent intensity of each atom without any interference between different atoms. Quite often the incoherent contribution is registered in the measurement as an unwanted background to the coherent intensity described by the second term of Equation (3).

\section{INELASTIG NEUTRON SGATTERING FROM ICE}

Recent work, which will be reviewed in this section, has analysed the coherent scattering from $\mathrm{D}_{2} \mathrm{O}\left(\mathrm{H}_{2} \mathrm{O}\right.$ has an overwhelming incoherent cross-section). The $\mathbf{U}_{j}$ in Equation (3) may or may not be time-dependent. If they are time-dependent, they may be diffusive with a relaxation time $\tau$ or oscillatory with a frequency $\nu$. In the oscillatory case a particular $\mathbf{U}_{j}$ is described by the sum over the amplitudes of all the lattice vibrations (phonons). The frequency-wavelength $(\nu-\lambda)$ relationship of the lattice vibrations gives the phonon dispersion curves. There are always $3 \mathcal{N}$ dispersion branches for each phonon wavevector $\mathbf{q}, \mathcal{N}$ is the number of atoms per unit cell. In ice Ih there are 36 branches as there are four molecules per unit cell.

Phonon dispersion curves have been reported by Renker (1973) who compared his experimental results with his lattice dynamics calculations. These calculations were based on the simplifying assumption that the mass of the $\mathrm{D}_{2} \mathrm{O}$ molecule is concentrated onto the 
oxygen site. The H-bonds are expressed in particular force constants. The model has only four particles per unit cell and hence calculates only 12 dispersion branches. The fit to the measured low-frequency branches was very good, because in these modes the molecules move as a whole. The branches not calculated were librations and intramolecular vibrations.

A lattice dynamics model with four real molecules per unit cell (Bosi and others, 1973) has been calculated for ordered protons (or orientationally-ordered molecules). This model predicts well-defined dispersion branches in the frequency region $240-300 \mathrm{~cm}^{-1}$, where librations and translations may couple to each other. This prediction will also have to be modified, because the orientational disorder is expected to broaden the librations, and, by coupling, the lattice modes as well.

Wong and others (1973), on the other hand, estimate that the interaction between static and transition dipoles should destroy the translational plane-wave modes above $240 \mathrm{~cm}^{-1}$. This means that collective excitations with well-defined dispersions should disappear above $240 \mathrm{~cm}^{-1}$ to be replaced by a broad band of excitations.

To clarify this problem Renker and Parisot (1976) performed an inelastic neutronscattering experiment on the hot-source three-axis spectrometer $\mathrm{IN}_{\mathrm{I}}$ of the Institut LaueLangevin. The results at 9o K (Fig. I) show two extremely flat phonon branches at 220 and $284 \mathrm{~cm}^{-1}$. The hatched regions indicate the width of the phonon groups which were broader than the experimental resolution. The flat dispersion could lead to the interpretation that they represent Einstein oscillations of single molecules. Renker and Parisot (1976) found intensity variations between one Brillouin zone in reciprocal space and the next. These intensity variations are produced by an eigenvector of the excitation within the unit cell. The authors conclude that these excitations have a short lifetime but within one oscillation the different atoms in the unit cell maintain amplitude and phase relations which are given by this eigenvector. The intensity variation could not be described by Renker's (1973) model which clearly states that the deuterium ions already play an important role in these modes. Above $4 \mathrm{IO} \mathrm{cm}^{-1}$ Renker and Parisot (I976) found a broad spectrum of librational excitations with weak maxima at $4 \mathrm{IO}$ and $480 \mathrm{~cm}^{-1}$. Apparently, it is the disorder or the lack of translational symmetry which does not allow collective librations of long lifetime.

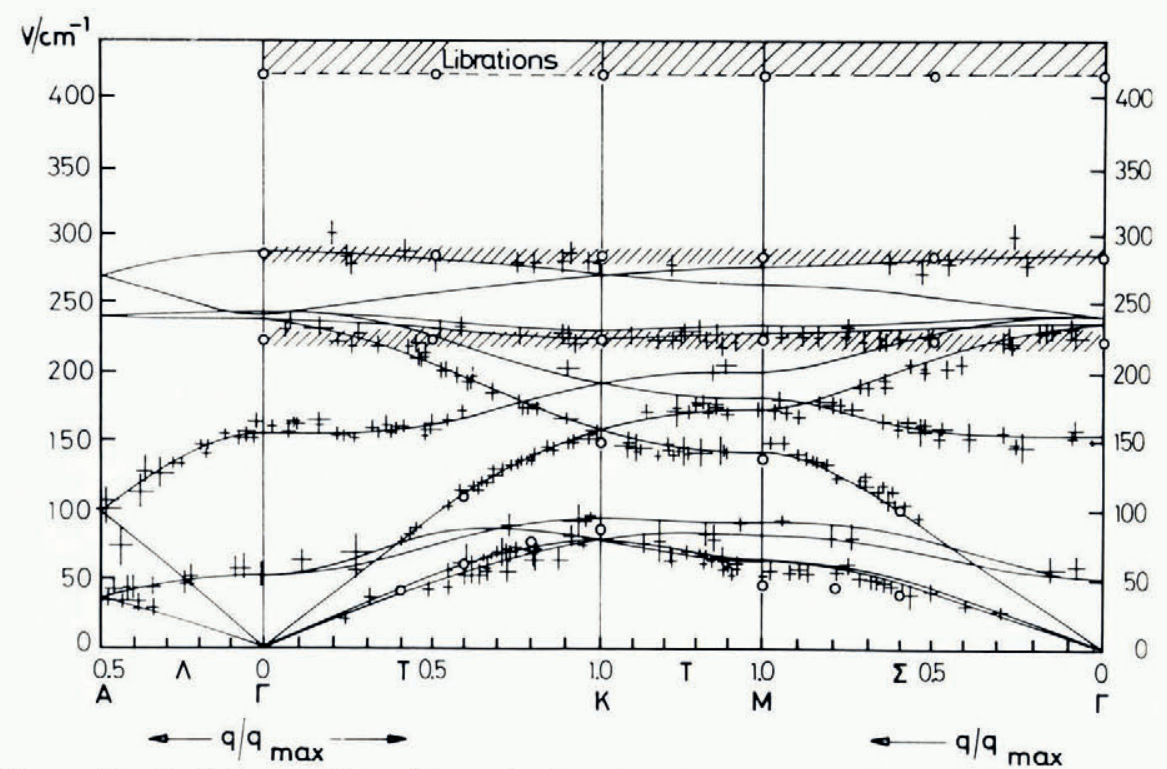

Fig. I. Phonon dispersion in hexagonal ice. New results from three-axis spectrometer (open circles) together with preliminary TOF data (crosses). The solid lines are a fit of a four-parameter valence force field to the data (Renker, 1976). 


\section{Diffuse NEUTRON SGATTERING}

As stated earlier the $\mathbf{U}_{j}$ 's in Equation (3) may or may not be time-dependent. For neutrons, resolution (estimation of the length of the neutron wavepacket) determines whether the time-dependence can be investigated or not. If $\mathbf{U}_{j}$ varies during the time in which the neutron wavepacket is passing by, then the time-dependence can be seen by inelastic scattering. The relaxation time $\tau$ of the proton disorder at $-10^{\circ} \mathrm{C}$ is about $10^{-5} \mathrm{~s}$ (Schmidt, 1973), which is much too long to be analysed at present.

The disorder of the protons in ice Ih described by their $\mathbf{U}_{j}$ 's gives rise to diffuse scattering, as shown by the coherent part of Equation (3), which is elastic as far as available resolution is concerned.

Villain and Schneider (1973) have presented model calculations and a few experimental results on $\mathrm{D}_{2} \mathrm{O}$, and $\mathrm{Kamb}$ ( 1973 ) reported preliminary results on $\mathrm{D}_{2} \mathrm{O}$ by Axe and Hamilton. Since then Schneider (unpublished) has collected many data relating to different sheets in reciprocal space. The data could be explained qualitatively by the model of Villain (1970). J. D. Axe (private communication) tried to Fourier transform his intensity distribution to obtain a correlation function of the $\mathbf{U}_{j}$ 's in real space, but the results were not satisfying. The major problem is the subtraction of the background. There is a considerable and unavoidable

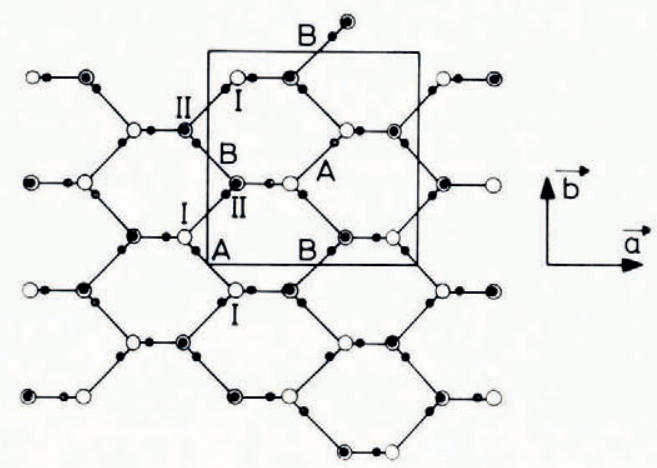

Fig. 2. Schematic structure of a water layer in copper formate tetrahydrate below $T_{0}$ (Youngblood and Axe, in press).

incoherent contribution everywhere and a coherent inelastic contribution from acoustic modes near the Bragg points. Close to the Bragg points, the knowledge of the diffuse intensity distribution is crucial as this intensity should vanish along symmetry directions due to the ice rule, as well as there being a small singularity at Bragg positions (Villain, 1970). Descamps and Coulon (1977) calculate the diffuse intensity distribution by the weak graph-expansion technique. The calculated patterns are very similar to those of Villain and Schneider (1973). Differences are in symmetry directions, along which Villain found vanishing intensity.

Recently Youngblood and Axe (in press) measured the diffuse neutron scattering in deuterated copper formate tetrahydrate (CFT). This substance contains layers of water molecules as seen schematically in Figure 2. Each layer may be considered as two-dimensional ice. Three out of four hydrogen bonds are in the layer and one is perpendicular (at site II). The protons (deuterons) are disordered above $248 \mathrm{~K}$ (phase transition temperature $T_{0}$ for deuterated CFT) and ordered below. Figure 2 gives the ordered structure where a ferroelectric polarization along $\mathbf{b}$ appears within one layer. The sign of the polarization alternates from layer to layer so that the three-dimensional order is antiferroelectric. The diffuse scattering (Fig. 3) above $T_{0}$ in the $H-K$ plane is essentially independent of $L$. The pattern is similar to ice Ih as the intensity disappears at the centres of the Brillouin zones. This is 


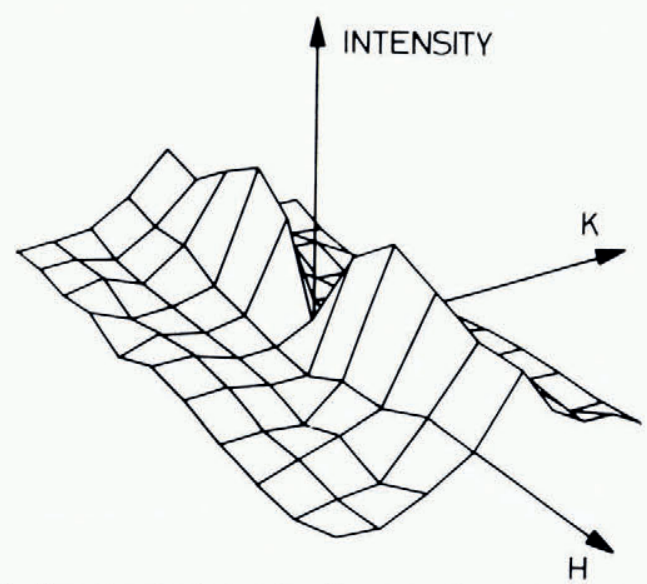

Fig. 3. Diffuse scattering intensity from disordered deuterium in copper formate tetrahydrate around $(0,4, I .5)$ at $26 I K$ ( roungblood and Axe, in press).

understood, as the polarization for $q=0$ is a conserved quantity due to the ice rules (Allen, I974). Below $T_{0}$, superlattice peaks appeared at $\left(H, K, L+\frac{1}{2}\right)$ and the diffuse scattering was almost invisible, indicating that the molecules had ordered. The absence of critical scattering at $\left(H, K, L+\frac{1}{2}\right)$ above $T_{0}$ is understood in terms of a dimer model (Youngblood, in press) which incorporates the ice rule. A correlation function for the dimers has not yet been calculated. Therefore the details of the intensity distribution have not yet been compared to predictions by models.

So far I have reported the recent neutron work on ice Ih which has come to my knowledge. This section has dealt with the coherent part of the scattering.

\section{The elastic incoherent STRUcture Factor}

For the rest of this paper I will discuss the information which one can get from the incoherent part of Equation (3), or more precisely from the interaction of the neutron with one particular proton. We will take the example of adamantane $\mathrm{C}_{10} \mathrm{H}_{16}$ which is a rather spherical molecule and performs rotational diffusion with a relaxation time $\tau$ in the high-temperature phase above $208 \mathrm{~K}$. In this case the $\mathbf{U}_{j}$ in Equation ( $\mathrm{I}$ ) for a particular proton is timedependent. The Fourier transform of the time dependence gives the energy dependence of the incoherent scattering law (Sköld, r968; Stockmeyer and Stiller, r968; Leadbetter and Lechner, in press)

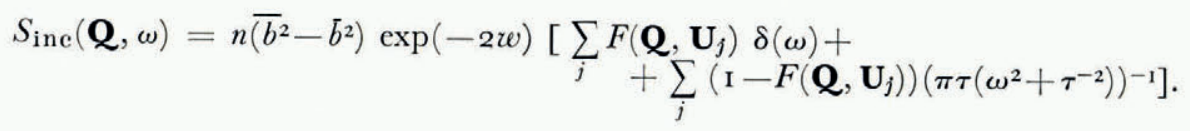

Both summations are carried out over the number of protons per molecule.

The function $F$ depends on $\mathbf{Q}$, on the orientation of $\mathbf{Q}$ to $\mathbf{U}_{j}$ and on the radius on which the proton $j$ is diffusing. If we integrate over $\omega$, we get

$$
S_{\text {inc }}(\mathbf{Q})=\int S_{\text {inc }}(\mathbf{Q}, \omega) \mathrm{d} \omega=n\left(\overline{b^{2}}-\bar{b}^{2}\right) \exp (-2 w) \mathcal{J},
$$

where $\mathcal{J}$ is the number of protons per molecule. 


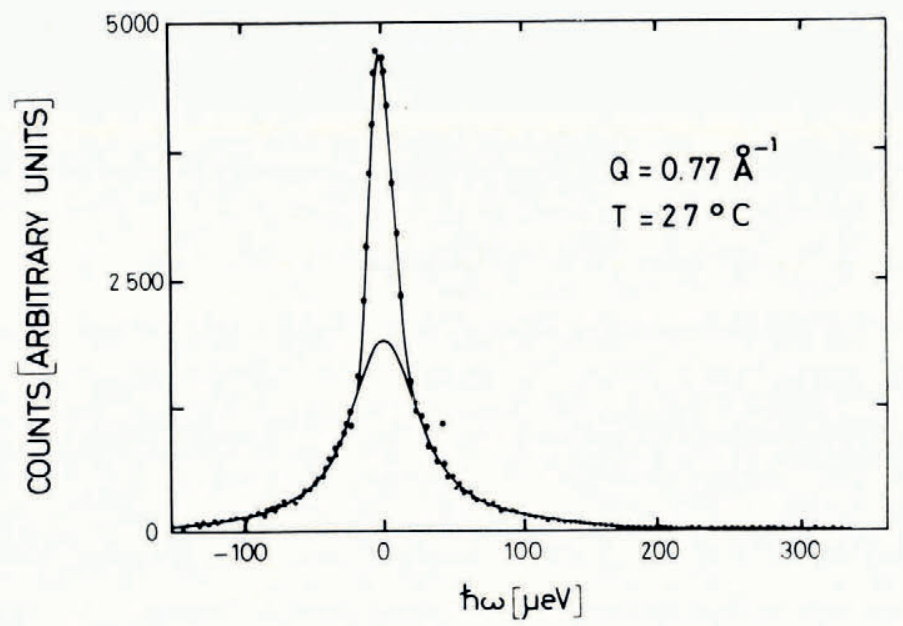

Fig. 4. High-energy resolution measurement on adamantane. The purely elastic part (Gaussian shape) appears on top of the inelastic (often called quasi-elastic) part (Lorentzian shape). The solid line is a computer fit to the data (Lechner and Heidemann, 1976$)$.

If $\tau$ is long and the experimental resolution too poor, then the integration is done immediately by the measurement and no $Q$-dependent structure can be seen. The wavepacket of the neutron is too short to see the movement of the proton. In ice Ih at $-10^{\circ} \mathrm{C}, \tau \approx 10^{-5} \mathrm{~s}$ (Schmidt, I973) which is too long to be resolved even by high-resolution instruments, but if the resolution is very good, one can separate the elastic part $(\delta(\omega)$ in Equation (4)) from the inelastic part (second part of Equation (5)). By the standards of neutron scattering, very high resolution is necessary to obtain data as seen in Figure 4 (Lechner and Heidemann, 1976). The elastic part (Gaussian, resolution of the instrument) appears on top of the inelastic part (Lorentzian). $\tau$ could be determined as a function of temperature (Lechner, 1976). At the moment, investigations are limited to $\tau<10^{-7} \mathrm{~s}$.

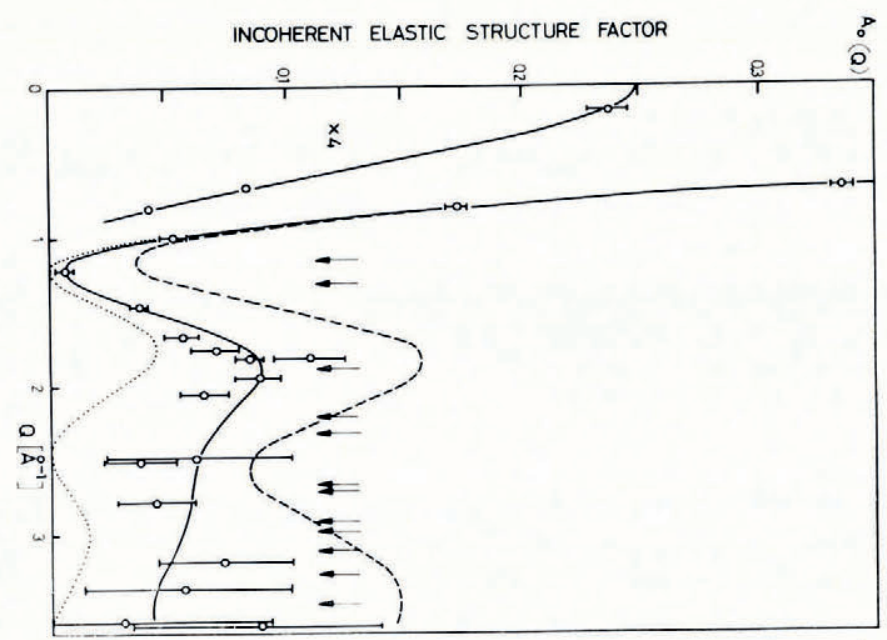

Fig. 5. Elastic incoherent structure factors in adamantane. $\bigcirc$ are experimental points with statistical error bars, ... rotational diffusion model, - four-fold rotation model, - - three-fold rotation model. Arrows indicate positions of Bragg peaks (Lechner and Heidemann, 1976). 
Interesting information is contained in the function $F$, the "elastic incoherent structure factor". This is the sum of the Fourier transforms of the density distribution of each single proton (which may be smeared over a sphere or distributed over several sites) if the molecule performs rotational jumps. The essential for obtaining purely elastic scattering is that the density stays finite for $t \rightarrow \infty$. In other words, the proton has to stay with the molecule and the molecule in the lattice. The same holds for a proton in a H-bond.

In the case of adamantane the open question concerned the kind of rotational diffusion. Was it continuous diffusion on a sphere, jump rotations around four-fold axes, or jump rotations around three-fold axes? The function $F$ was calculated for the three models and compared with the measured $F$ (Fig. 5) (Lechner and Heidemann, I976). It could be concluded that the adamantane molecule performs jump diffusion around four-fold axes.

Besides the mentioned length of $\tau$, another difficulty arises if the sites over which the proton is smeared are close together, because in this case the interference pattern in $F$ appears at large momentum transfer $|\mathbf{Q}|$, which is difficult to obtain together with high-energy resolution.

\section{TUNNELLING BETWEEN ROTATIONAL GROUND STATES}

The incoherent scattering from a proton arises because the scattering lengths depend on the neutron-proton spin configuration, i.e. parallel or antiparallel. In an unpolarized proton system one-third of the incoherent scattering takes place without spin flip, and two-thirds with spin flip. For molecules containing protons this means that an incoherent scattering process can change the total nuclear spin $I$ of the molecule by $\Delta I=0, \pm \mathrm{r}$. The energy levels for rotational motion of molecules depend generally on the total nuclear spin $I$. A well-known example is that of para- and ortho-hydrogen. In the neutron-scattering experiment one will obtain energy transfers associated with $\Delta I= \pm \mathrm{I}$ and with $\Delta I=\mathrm{o}$ if different states, for the same total spin $I$, are not degenerate (Alefeld and others, 1975; Press and Kollmar, 1975; Hüller, I977). From the transition energies the molecular-level scheme, and in simple cases the corresponding rotational potential, can be derived.

The tunnel splitting of the $\mathrm{NH}_{4}$-librational groundstate in $\mathrm{NH}_{4} \mathrm{ClO}_{4}$ has been measured by high-resolution inelastic neutron scattering by Prager and others (1976). Figure 6 shows the energy transfers in gain and loss at $5 \mathrm{~K}$. Energy gain and loss is equally probable as these

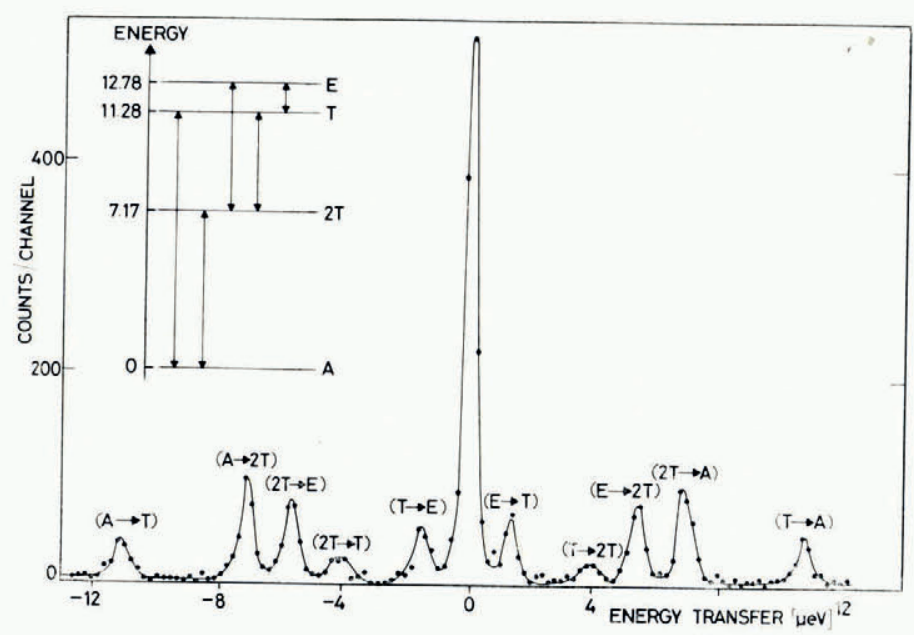

Fig. 6. High-energy resolution spectrum of $\mathrm{NH}_{4} \mathrm{ClO}_{4}$ at ${ }_{5} \mathrm{~K}$. A derized level scheme is inserted, where $\mathrm{A}$ has the total spin $I=2, T$ has $I=1$, and $E$ has $I=O$ (Prager and others, 1976 ). 


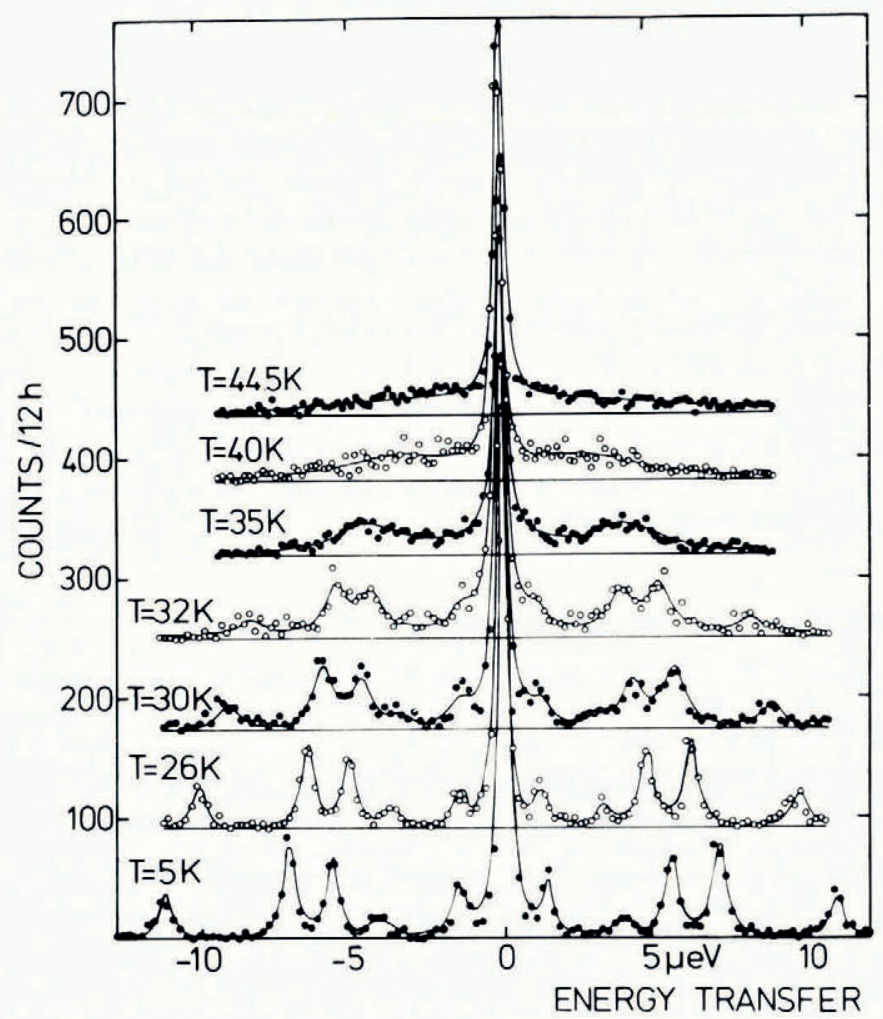

Fig. 7. High-energy resolution spectra of $\mathrm{NH}_{4} \mathrm{ClO}_{4}$ at various temperatures (Prager and others, ${ }^{1976} 6$ ).

low energies are still small compared with temperature. The level scheme indicates the transitions. From the measurements it is concluded that the potential of the $\mathrm{NH}_{4}{ }^{+}$ion in $\mathrm{NH}_{4} \mathrm{ClO}_{4}$ is three-dimensional. The splitting of the $T$ levels $(I=\mathrm{I})$ shows that the rotational potential is of a symmetry lower than tetrahedral.

With increasing temperature the quantum-mechanical tunnel splitting should go over into the classical rotational-jump diffusion, which was discussed earlier. The effect is observed in $\mathrm{NH}_{4} \mathrm{ClO}_{4}$ (Prager and others, 1976) and shown in Figure 7. With increasing temperature the tunnel lines shift towards smaller energies and their width increases. The width of the tunnel lines follows an Arrhenius law and corresponds at higher temperatures to the relaxation time $\tau$ for rotational-jump diffusion.

\section{Conclusion}

Recent neutron scattering measurements on ice Ih and on two-dimensional ice have been reported. The progress since the Ottawa conference is not great; it simply reflects the fact that translational symmetry, which normally eases experimental and theoretical work into lattice dynamics, is absent in ice.

In addition, I have presented the results of investigations into the protonic positions and movements in solids. Such investigations involve the use of high-resolution inelastic neutron scattering. This might challenge one or other participants of this conference to use the facilities of the ILL. Unfortunately, however, applications of these techniques to ice still seem improbable. 


\section{Acknowledgements}

I wish to thank Drs J. D. Axe, A. Heidemann, R. Lechner, B. Renker, and R. Youngblood for helpful discussions.

\section{REFERENGES}

Alefeld, B., and others. I975. The one-dimensional $\mathrm{CH}_{3}$-quantum rotator in solid 4-methyl-pyridine studied by inelastic neutron scattering, [by] B. Alefeld, A. Kollmar and B. A. Dasannacharya. Fournal of Chemical Physics, Vol. 63 , No. 10, p. 4415-17.

Allen, G. R. r 974 . Dimer models for the antiferroelectric transition in copper formate tetrahydrate. Fournal of Chemical Physics, Vol. 6o, No. 8, p. 3299-309.

Bosi, P., and others. 1973. Lattice dynamics of hydrogen-bonded crystals: ice Ih, [by] P. Bosi, R. Tubino and G. Zerbi. (In Whalley, E., and others, ed. Physics and chemistry of ice: papers presented at the Symposium on the Physics and Chemistry of Ice, held in Ottawa, Canada, 14-18 August 1972. Edited by E. Whalley, S. J. Jones, L. W. Gold. Ottawa, Royal Society of Canada, p. 98-ro2.)

Descamps, M., and Coulon, G. 1977. Series expansion calculations of the elastic neutron diffuse scattering: ice Ih. Chemical Physics, Vol. 25, No. 1, p. 170-79.

Dorner, B., and Comès, R. I977. Phonons and structural phase transformations. (In Lovesey, S. W., and Springer, T., ed. Dynamics of solids and liquids by neutron scattering. Berlin, etc., Springer-Verlag, p. 127-96. (Topics in Current Physics, Vol. 3.))

Hüller, A. 1977. Rotational tunneling in solids: the theory of neutron scattering. Physical Review B, Third Ser., Vol. 16 , No. 5, p. $1844-57$.

Kamb, W. B. 1973. Crystallography of ice. (In Whalley, E., and others, ed. Physics and chemistry of ice: papers presented at the Symposium on the Physics and Chemistry of Ice, held in Ottawa, Canada, 14-18 August 1972. Edited by E. Whalley, S. F. Fones, L. W. Gold. Ottawa, Royal Society of Canada, p. 28-41.)

Leadbetter, A. J., and Lechner, R. E. In press. Neutron scattering studies. (In Sherwood, J. N., ed. The plastic crystalline state.)

Lechner, R. E. 1976. Rotational motion in plastic-crystalline adamantane: results from high resolution quasielastic neutron scattering. (In Moon, R. M., ed. Proceedings of the Conference on Neutron Scattering [held in] Gatlinberg, Tennessee, U.S.A., June 6-10, 1976 . Oak Ridge, Tennessee, Oak Ridge National Laboratory, Vol. I, p, 310-16.)

Lechner, R. E., and Heidemann, A. 1976. Rotational motion in plastic adamantane: the elastic incoherent structure factor. Communications on Physics, Vol. 1, No. 7, p. 21 $3^{-2} \mathrm{I}$.

Prager, M., and others. 1976 . The tunnel splitting of the $\mathrm{NH}_{4}$-librational groundstate in $\mathrm{NH}_{4} \mathrm{ClO}_{4}$ measured by inelastic neutron scattering, by M. Prager, B. Alefeld and A. Heidemann. (In Brunner, H., and others, ed. Magnetic resonance and related phenomena. Proceedings of the XIXth Congress Amperre, Heidelberg, September 1976. Edited by H. Brunner, K. H. Hausser and D. Schweitzer. Heidelberg, Geneva, Groupement Ampère, p. 389-94.)

Press, W.. and Kollmar, A. 1975. $\mathrm{CH}_{4}$ : tunneling states, rotations and phase transition in a quantum molecular crystal. Solid State Communications, Vol. i 7, No. 4, p. 405-08.

Renker, B. 1973. Lattice dynamics of hexagonal ice. (In Whalley, E., and others, ed. Physics and chemistry of ice: papers presented at the Symposium on the Physics and Chemistry of Ice, held in Ottawa, Canada, I4-18 August 1972. Edited by E. Whalley, S. F. Jones, L. W. Gold. Ottawa, Royal Society of Canada, p. 82-86.)

Renker, B., and Parisot, G. 1976. Lattice dynamics of hexagonal ice. Annex to the Annual Report, Institut Max von Laue - Paul Langevin (Grenoble), I 976, p. 69-7o.

Schmidt, V. H. r973. Deuteron n.m.r. relaxation in $\mathrm{D}_{2} \mathrm{O}$ ice Ih. (In Whalley, E., and others, ed. Physics and chemistry of ice: papers presented at the Symposium on the Physics and Chemistry of Ice, held in Ottawa, Canada, I4-18 August 1972. Edited by E. Whalley, S. F. Jones, L. W. Gold. Ottawa, Royal Society of Canada, p. 21 2-17.)

Schneider, J. Unpublished. Elastisch diffuse Neutronenstreuung an Eis Ih. [Dr. rer. nat. thesis, Technische Universität München, I975.]

Sköld, K. J. ig68. Effects of molecular orientation in solid methane on the quasielastic scattering of thermal neutrons. Journal of Chemical Physics, Vol. 49, No. 5, p. 2443-45.

Stockmeyer, R., and Stiller, H. 1968. Phonons, torsons and rotational diffusion in adamantane. Physica Status Solidi, Vol. 27, No. I, p. 269-80.

Villain, J. I970. The 3-dimensional eight vertex model and the proton-proton correlation function in ice. Solid State Communications, Vol. ıo, No. 10, p. 967-70.

Villain, J., and Schneider, J. 1973. Correlation functions in nonpolar ice. (In Whalley, E., and others, ed. Physics and chemistry of ice: papers presented at the Symposium on the Physics and Chemistry of Ice, held in Ottawa, Canada, $14^{-18}$ August 1972. Edited by E. Whalley, S. 7. Jones, L. W. Gold. Ottawa, Royal Society of Canada, p. 285-90.)

Wong, P. T. T., and others. I973. The Raman spectrum of the translational lattice vibrations of ice Ih, [by] P. T. T. Wong, D. D. Klug and E. Whalley. (In Whalley, E., and others, ed. Physics and chemistry of ice: papers presented at the Symposium on the Physics and Chemistry of Ice, held in Ottawa, Canada, I4-18 August I972. Edited by E. Whalley, S. 7. Jones, L. W. Gold. Ottawa, Royal Society of Canada, p. 87-92.)

Youngblood, R. In press. Fluctuations in two-dimensional ice. Physical Review B.

Youngblood, R., and Axe, J. D. In press. Neutron scattering study of short-range order in copper formate tetrahydrate. Ferroelectrics. 


\section{DISGUSSION}

J. F. NAGLE: I would just like to amplify the remarks concerning Youngblood's work on copper formate tetrahydrate (CFT). Although it has not yet been possible to compute scattering amplitudes for the Allen model, Youngblood did use the exact correlation functions of Fisher and Stephenson for the dimer model, which is the high-temperature limit of the Allen model, to compute scattering amplitudes which display the $\mathrm{V}$-shaped minimum. This is very strong evidence in support of ice rules in CFT. Thus, it is fair to say that a substantial amount of theoretical analysis has already been completed and shows good agreement with experiment.

B. Dorner: This is correct. I did not go into a detailed review of Youngblood's work. As far as the measured intensity distribution in reciprocal space is concerned, there is no calculated distribution for larger $q$ 's. 